\title{
An Examination of Racial Differences in Process and Outcome of Colorectal Cancer Care Quality Among Users of the Veterans Affairs Health Care System
}

\author{
Leah L. Zullig ${ }^{1,2}$, George L. Jackson ${ }^{1,3}$, Morris Weinberger ${ }^{1,2}$, Dawn Provenzale ${ }^{1,4}$, Bryce B. \\ Reeve $^{2}$, and William R. Carpenter ${ }^{2}$ \\ ${ }^{1}$ Center of Excellence for Health Services Research in Primary Care, Durham Veterans Affairs \\ Medical Center, Durham, NC \\ 2Department of Health Policy and Management, University of North Carolina at Chapel Hill, \\ Chapel Hill, NC \\ ${ }^{3}$ Division of General Internal Medicine, Duke University, Durham, NC \\ ${ }^{4}$ Division of Gastroenterology, Duke University, Durham, NC
}

\begin{abstract}
Our objective was to examine potential racial differences in CRC care timeliness in the Veterans Affairs (VA) health care system. Using a national sample of white and African American men, we examined time from surgery to (1) adjuvant chemotherapy initiation (stages II-III disease); (2) surveillance colonoscopy (stages I-III disease), and (3) death (stages I-III disease). We identified no clinically meaningful racial differences in CRC care timeliness.
\end{abstract}

Background-Veterans Affairs (VA) manages the largest US integrated health care system. Although quality of VA colorectal cancer (CRC) care is well chronicled, there is a paucity of research examining racial differences in this care. This study examines racial differences in 2 dimensions of quality of VA CRC care: processes (time to treatment) and outcomes (survival).

Patients and Methods-Retrospective data were from the VA External Peer Review Program (EPRP), a nationwide VA quality-monitoring program. Study patients were white and African American men diagnosed with nonmetastatic CRC between 2003 and 2006 who received definitive CRC surgery. We examined 3 quality indicators: time from (1) surgery to initiation of adjuvant chemotherapy (stages II-III disease), (2) surgery to surveillance colonoscopy (stages I-III disease), and (3) surgery to death (stages I-III disease). Unadjusted analyses used log-rank and Wilcoxon tests. Adjusted analyses used Cox proportional hazard models.

Results-In unadjusted analyses, there was no evidence of racial differences across the 3 quality measures. In adjusted Cox regression, there were no racial differences in time to initiation of chemotherapy (hazard ratio [HR], $0.82 ; P=.61$ ) or surgery to death (HR, $0.94 ; P=.49$ ). In adjusted Cox regression, among those receiving colonoscopy within 7 to 18 months after surgery, white patients experienced slightly shorter median times to surveillance colonoscopy than did African American patients ( 367 vs. 383 days; HR, 0.63; $P=.02$ ). 
Conclusion-Other than a small racial difference in timing of surveillance colonoscopy, there was little evidence of racial differences in quality of CRC care among VA health care system users.

\section{Keywords}

Colorectal neoplasms; Health care disparities; Health care quality; Minority health; US Veterans Affairs

\section{Introduction}

The Department of Veterans Affairs (VA) manages the largest integrated health care system in the United States, treating approximately $3 \%$ of patients with cancer. ${ }^{1}$ Since its national reorganization and transformation in the mid-1990s, ${ }^{2}$ the VA has been a leader in providing high-quality equitable care. The quality of cancer care provided in the VA has been extensively examined, with the VA generally performing equal to or better than the private sector. ${ }^{3,4}$ Quality of colorectal cancer (CRC) care in particular has been lauded as an area in which the VA provides excellent care. ${ }^{3,4}$ In addition to providing quality care overall, there is evidence that racial disparities in CRC care quality may be less significant in the VA than in nonfederal health care systems. ${ }^{5-7}$

An important process measure reflecting quality care is timeliness in receiving evidencebased treatments. The VA has long-standing processes for quality monitoring and performance improvement, focused on achieving guideline-concordant clinical care, which may support its success as a leader in the provision of quality care. ${ }^{8-11}$ There is a systemwide emphasis on adhering to guidelines based on strong scientific and clinical evidence. However, standards for timeliness of care are largely consensus based. Perhaps as a result, timeliness standards have not been widely implemented in the VA or other large integrated health care systems. The VA has evaluated several CRC interventions and collaborative efforts to address delays in diagnosis and follow-up on positive screening test results. ${ }^{12-14}$ Less attention has been focused on timeliness of care during CRC treatment and early surveillance phases.

Survival can be considered an outcome measure of quality. Although several studies examining survival have produced mixed results about the presence of racial differences, ${ }^{6,15,16}$ there is a paucity of literature describing racial differences in timeliness of VA CRC care. Landrum et al. compared cancer-specific and all-cause mortality rates for men older than 65 years receiving care in the VA vs. fee-for-service Medicare. Compared with similar fee-for-service Medicare patients, survival rates for VA users with CRC were equal or better. ${ }^{3}$ In the 1990s, Dominitz et al. examined potential racial differences in CRC survival rates, finding similar relative 5-year survival rates for African American and white patients with CRC seeking care in the VA health care system. ${ }^{6}$ Jackson et al. examined the timeliness of treatment for patients with nonmetastatic CRC in the VA health care system. For patients with stage II and stage III disease, they found a median of 20 days between diagnosis and initiation of CRC treatment. There was a median of 50 days between definitive surgical resection and start of adjuvant chemotherapy. ${ }^{17}$ The authors did not examine patient or disease characteristics associated with timeliness of care.

We expand previous work by examining racial differences in 2 dimensions of quality of VA CRC care: processes (time to treatment) and outcomes (survival). 


\section{Patients and Methods}

\section{Data Source and Patient Sample}

The VA External Peer Review Program (EPRP), the national program for assessing quality of VA health care, was the primary data source. ${ }^{18}$ In 2007, the Veterans Health Administration Office of Analytics and Business Intelligence (formerly known as the Office of Quality and Performance) oversaw a national medical record abstraction effort to assess the quality of CRC care. Abstractors accessed the electronic health record remotely, collecting data on disease characteristics and health care delivery provided to patients throughout the VA health care system. We augmented EPRP data with clinical comorbidity and demographic information contained in the Veterans Affairs Central Cancer Registry and administrative data (specifically inpatient and outpatient Medical SAS files).

The sample has previously been described in detail. ${ }^{17}$ Succinctly, patients were identified for inclusion in EPRP based on a search algorithm that defined a representative sample of VA patients diagnosed with CRC between October 1, 2003 and March 31, 2006. The algorithm made use of administrative diagnosis, procedure, and encounter data stored in the centralized VA Decision Support System. ${ }^{17,19}$ Eligible patients had an ICD-9 code for colon and/or rectal cancer within 3 months (before or after) of the study diagnosis period. ${ }^{20}$ Eligible patients must also have had a clinic visit, surgical procedure, or pathology report in the VA that corresponded to receipt of medical services within the aforementioned time frame. The final analytic data set included patients with nonmetastatic CRC (stages I-III), an incident occurrence (first diagnosis of CRC occurred during the study period), receipt of definitive surgical resection for CRC, and a successful link with information from VA administrative data sources. We obtained vital status information in May 2012, resulting in a median follow-up period of approximately 6 years (2160.5 days) after surgery. Because of the small number of non-African American minorities and women, analysis was restricted to white and African American male patients (Fig. 1). We did not have information regarding Hispanic ethnicity.

\section{Measures}

We examined 3 stage-specific quality metrics for CRC care: (1) time from definitive CRC surgical resection to initiation of 5-fluorouracil (5-FU)-based adjuvant (eg, postoperative) chemotherapy (stage II or III), ${ }^{21}$ (2) time from definitive surgical resection to receipt of surveillance colonoscopy (stage I, II, or III), ${ }^{22-27}$ and (3) time from definitive CRC surgical resection to death (stage I, II, or III). The first 2 measures reflect process indicators of quality, whereas the third indicator measures survival as a critical outcome. We also examined whether comorbidities and demographic factors were mediators of differences in survival and other timeliness of care events.

The date of surgical resection was used as the anchor date for all 3 measures for several reasons. First, the date of surgery is a decisive date, unlike the date of diagnosis, which is often difficult to define (eg, date of positive screening test result, date of pathology report, date of physician's suspicion of CRC). Exploratory analysis of these data confirmed that for many patients, the date of surgery preceded the date of diagnosis, likely because of ambiguity about the date of diagnosis. Second, all patients in the sample underwent surgical resection, making it a feasible anchor date. Finally, some patients may have been diagnosed outside of the VA health care system but then entered the VA health care system for their cancer care. Using the surgery date as the index enables a better examination of processes of care within the VA.

Additional inclusion and exclusion criteria were applied for each of the 3 quality measures. For surveillance colonoscopy, patients must have survived at least 1 year after surgery to be 
included in analysis. Most clinical guidelines during this period, including the National Comprehensive Cancer Network ${ }^{26,27}$ and the American Cancer Society, ${ }^{28}$ recommended a surveillance colonoscopy 1 year after surgery. For descriptive and univariate analyses, we included all surveillance colonoscopy results in the analysis. For multivariable regression analysis, we limited surveillance colonoscopies to those occurring within 7 to 18 months after surgery, because colonoscopies occurring before 7 months after surgery might be diagnostic; the maximum of 18 months reflects pragmatic challenges to receiving this test within the recommended 12 months (eg, scheduling challenges, patient preference). For each of the 3 quality indicators, we calculated the number of days between the surgery date and the date of the event.

Covariates, identified a priori, included patient characteristics associated with timeliness of cancer care. ${ }^{29-31}$ We considered both demographic (age at diagnosis, marital status, geographic region) and disease (stage at diagnosis, comorbidity) characteristics. The comorbidity measure was the National Cancer Institute (NCI) Combined Comorbidity score, created from inpatient and outpatient medical record data from 1 year before CRC diagnosis until 1 month before diagnosis. This weighted comorbidity score has previously been validated in a CRC cohort. $^{32}$

\section{Statistical Analysis}

We used the Kaplan-Meier method to estimate time-to-event curves. To compare differences in unadjusted survival curves, we used the log-rank and Wilcoxon tests. Multivariate Cox proportional hazards models were used to assess the prognostic power of race for time to event in the presence of the aforementioned covariates. The Efron method was used to handle ties. ${ }^{33,34}$ Data management and analyses were conducted in STATA, version 11 (StataCorp LP, College Station, TX) and SAS, version 9.2 (SAS Institute, Cary, NC). The institutional review boards at the Durham VA Medical Center and at the University of North Carolina at Chapel Hill approved this project.

\section{Results}

The final sample consisted of 2022 men who met eligibility criteria (Fig 1). Reflecting the overall VA patient population, the sample had a mean age at diagnosis of 68 years. The majority of patients were white (85\%), married (52\%), and lived in the southern United States (38\%). Disease stage was approximately evenly distributed. The mean National Cancer Institute (NCI) Combined Comorbidity Index score was 0.27 (Table 1). The most commonly diagnosed individual comorbid conditions were diabetes $(27 \%)$ and cardiovascular-related diseases (24\%).

Across the sample, the median time from surgery to initiation of adjuvant 5-FU-based chemotherapy was 49 days; medians were not statistically different for African American and white patients (55 vs. 49 days, respectively; $P=.71$ ). In unadjusted analyses, there were no statistically significant racial differences in time-to-event curves for surgery to initiation of adjuvant 5-FU-based chemotherapy (Wilcoxon $P=.78$; $\log$-rank $P=.10$ ). Similarly, in adjusted multivariable Cox regression, race was not associated with time from surgery to start of adjuvant $5-\mathrm{FU}-$ based chemotherapy (HR, $0.82 ; P=.61$ ). The region in which patients received care was significant. Compared with those living in the southern United States, patients living in the northern (HR, 0.06; $P=.01)$ and central $(\mathrm{HR}, 0.33 ; P=0.04)$ regions had shorter times from surgery to chemotherapy. Small sample sizes did not permit us to explore race by region interactions.

When examining all surveillance colonoscopies after surgical resection, the mean time from surgery to first surveillance colonoscopy was 396 days; the median time was 368 days. 
Unadjusted analyses found no statically significant differences in medians between African American and white patients (383 vs. 367 days; $P=.10$ ) or in time-to-event curves for time from surgery to first surveillance colonoscopy (Wilcoxon $P=.23$; log-rank $P=.05$ ). In adjusted multivariable regression analyses, considering only surveillance colonoscopies within a guideline-concordant 7 to 18 months after surgery, a racial difference in time to receipt of first surveillance colonoscopy was suggested. White race was protective for a shorter time to surveillance colonoscopy (HR, 0.63; $P=.02$ ) (Table 2). Although the association was statistically significant, the magnitude of the difference is small (16 days) and not likely to be clinically meaningful. Compared with patients living in the southern United States, those living in the central region (HR, 1.84; $P=0.00$ ) had longer times from surgery to colonoscopy. Compared with patients aged 75 years or older, patients (HR, 1.60; $P=.04$ ) aged 65 to 74 years were more likely to receive surveillance colonoscopy.

Across the sample, the median time from surgery to death was 1053 days, and the unadjusted difference was similar for African American and white patients (1050 vs. 1062 days, respectively; $P=.04)$. In unadjusted analyses, there were no statistically significant racial differences in time-to-event curves in time from surgery to death (Wilcoxon $P=.32$; $\log$-rank $P=.33$ ).

Similarly, in adjusted multivariable Cox regression, race was not associated with time from surgery to death (HR, $0.94 ; P=.49)$. Notably, several covariates were significant. Patients aged 55 years or younger had a lower hazard of death compared with patients 75 years or older (HR, 0.76; $P=.04)$. Compared with patients with stage II disease, those with stage I disease had reduced hazards of death (HR, $0.84 ; P=.04)$ and those with stage III disease had increased hazards of death (HR, $1.32 ; P=.00)$, which would be expected based on known clinical outcomes for cancer stage (Table 2).

\section{Discussion}

We examined whether racial differences existed in the quality of CRC care delivered by the VA, the largest integrated health care system in the United States. Using 2 stage-specific, evidence-based process measures (time to adjuvant chemotherapy and time to colonoscopy) and patient outcome (survival after surgery), we found no meaningful racial disparities with respect to these 3 quality measures. These findings support the perception of the VA as an "equal access system" committed to the provision of quality timely CRC care. ${ }^{2,16,35}$

The first process quality measure examined was time from surgery to initiation of 5-FUbased adjuvant chemotherapy. Consistent with previous literature, we found that the VA provides racially equal care on this metric. ${ }^{6}$ We identified possible geographic variations in care. Because of the sample size, this analysis aggregated regional data at a relatively high level (eg, 4 geographic regions nationwide). Based on these data, we do not know whether patients of different races who are receiving health care in the VA system disproportionately live in specific geographic regions. It is possible that these regional differences confound the ability to accurately assess racial differences. Future analyses could further investigate this phenomenon using more granular regional data.

In our analyses, potential racial differences were identified for only 1 process measurereceipt of surveillance colonoscopy. The difference in elapsed time to colonoscopy between the 2 groups (approximately 16 days on average) is small and the clinical significance of this difference is likely minimal. To ensure that this gap does not widen, additional research is needed to understand underlying mechanisms creating this time difference. Although not the focus of our analyses, there is nearly universal evidence that CRC survivors receive inadequate colonoscopic surveillance. The under-provision of surveillance colonoscopy has 
been documented among Medicare users, population-based patient samples, and users of the VA health care system. ${ }^{17,22,29,31}$ Although surveillance colonoscopy is generally underreceived, previous studies have suggested that patients of white and African American races receive colonoscopy equitably within the VA health care system. ${ }^{6}$ This is important contextual information for interpreting our study findings. This finding is consistent with previous studies on the subject. A recent systematic literature review by Salz et al. examined differences in timeliness of colonoscopy use among CRC survivors receiving care at multiple types of health care institutions. ${ }^{22}$ The authors identified 8 studies addressing racial differences in time to colonoscopy. Half of the articles described a small but significant racial difference in receipt of timely colonoscopy; the remaining half showed a nonsignificant trend in the same direction. ${ }^{22}$ There is no scientific evidence suggesting that a narrow difference in time (eg, 16 days on average) would impact care quality or patient outcome. There were also differences in age at diagnosis and receipt of surveillance colonoscopy; older people were less likely to receive a timely colonoscopy than their younger counterparts. This is as anticipated. Increasing age is often associated with decreased performance status and increased comorbidity burden. Therefore, it is possible that few patients aged 75 years or older would be expected to receive surveillance colonoscopy, and for those who do receive surveillance colonoscopy, the timeline may be extended.

As an outcome of care quality, we examined postsurgical survival times. Among the $49 \%$ who died $(\mathrm{n}=992)$, patients survived a median of approximately 3 years after surgery. Although we controlled for comorbidity status, this survival time for patients with nonmetastatic disease may be evidence of patient frailty. This survival finding may impact receipt of surveillance care (eg, a high degree of patient frailty could explain the appropriate withholding of surveillance colonoscopy). We identified similar survival times among different racial groups. In nonfederal health care settings, including integrated systems serving Medicare patients, there have been mixed findings regarding racial differences in CRC survival. ${ }^{6,36-38}$ Our finding of similar postsurgical survival rates between white and African American patients may suggest that the VA provides similar processes of quality care to patients throughout their disease trajectory, translating into comparable survival times. This hypothesis is supported by existing literature. ${ }^{17,38}$

This analysis has several limitations. First, veterans who receive care through the VA health care system have greater comorbidity than the general population. ${ }^{39}$ We adjusted for comorbidity, but some patients may have appropriately not received care (eg, too frail to undergo colonoscopy). Our analysis was limited to men and those receiving care in the VA. Because of the small number of women in these data (43 overall; $88.4 \%$ white and $11.6 \%$ African American), we were unable to make statistical inferences about this group. The number of women seeking care in the VA health care system is growing, and future studies should endeavor to assess cancer care timeliness in this group. Some patients receiving care in the VA health care system may also receive a portion of their cancer care elsewhere. Future research should endeavor to include information from multiple data sources. Despite these limitations, our analysis provides important insight into the quality and timeliness of VA CRC care.

\section{Conclusion}

We assessed key process and outcome measures of care quality and observed no evidence of clinically meaningful racial differences in timeliness of CRC care provided by the VA health care system. This may be a testament to the VA's history as an "equal access system"2,16,35 and its established commitment to ongoing quality monitoring and improvement. 


\section{Acknowledgments}

The authors acknowledge the data set preparation provided by David H. Abbott at the Durham VA Medical Center.

This study was supported by the Durham, NC Veterans Affairs (VA) Medical Center Health Services Research \& Development (HSR\&D) Center of Excellence. Development of the data set was funded by funds transfer from the Veterans Health Administration Office of Quality and Performance to the HSR\&D Center of Excellence at the Durham, NC, VA Medical Center. Dr Zullig was funded by the National Cancer Institute (5R25CA116339). Dr. Weinberger is a VA HSR\&D Senior Research Career Scientist (RCS 91-408).

\section{References}

1. Zullig LL, Jackson GL, Dorn RA, et al. Cancer incidence among patients of the U.S. Veterans Affairs Health Care System. Mil Med. 2012; 177:693-701. [PubMed: 22730846]

2. Kizer KW, Dudley RA. Extreme makeover: transformation of the veterans health care system. Annu Rev Public Health. 2009; 30:313-39. [PubMed: 19296778]

3. Landrum MB, Keating NL, Lamont EB, et al. Survival of Older Patients with cancer in the Veterans Health Administration versus fee-for-service Medicare. J Clin Oncol. 2012; 30:1072-9. [PubMed: 22393093]

4. Keating NL, Landrum MB, Lamont EB, et al. Quality of care for older patients with cancer in the Veterans Health Administration versus the private sector: a cohort study. Ann Intern Med. 2011; 154:727-36. [PubMed: 21646556]

5. Alexander DD, Waterbor J, Hughes T, Funkhouser E, Grizzle W, Manne U. African-American and Caucasian disparities in colorectal cancer mortality and survival by data source: an epidemiologic review. Cancer Biomark. 2007; 3:301-13. [PubMed: 18048968]

6. Dominitz JA, Samsa GP, Landsman P, Provenzale D. Race, treatment, and survival among colorectal carcinoma patients in an equal-access medical system. Cancer. 1998; 82:2312-20. [PubMed: 9635522]

7. Landrum MB, Keating NL, Lamont EB, Bozeman SR, McNeil BJ. Reasons for underuse of recommended therapies for colorectal and lung cancer in the Veterans Health Administration. Cancer. 2012; 118:3345-55. [PubMed: 22072536]

8. Francis J, Perlin JB. Improving performance through knowledge translation in the Veterans Health Administration. J Contin Educ Health Prof. 2006; 26:63-71. [PubMed: 16557507]

9. McQueen L, Mittman BS, Demakis JG. Overview of the Veterans Health Administration (VHA) Quality Enhancement Research Initiative (QUERI). J Am Med Inform Assoc. 2004; 11:339-43. [PubMed: 15187071]

10. Trivedi AN, Grebla RC. Quality and equity of care in the veterans affairs health-care system and in medicare advantage health plans. Med Care. 2011; 49:560-8. [PubMed: 21422951]

11. Trivedi AN, Matula S, Miake-Lye I, Glassman PA, Shekelle P, Asch S. Systematic review: comparison of the quality of medical care in Veterans Affairs and non-Veterans Affairs settings. Med Care. 2011; 49:76-88. [PubMed: 20966778]

12. Fisher DA, Zullig LL, Grambow SC, et al. Determinants of medical system delay in the diagnosis of colorectal cancer within the Veteran Affairs Health System. Dig Dis Sci. 2010; 55:1434-41. [PubMed: 20238248]

13. Partin MR, Powell AA, Nugent S, Ordin DL. Colorectal cancer diagnosis improvement project evaluation demonstrates the importance of using multiple measures to track progress toward timeliness goals. J Healthc Qual. 2013; 35:41-8. [PubMed: 22192595]

14. Powell AA, Gravely AA, Ordin DL, Schlosser JE, Partin MR. Timely follow-up of positive fecal occult blood tests strategies associated with improvement. Am J Prev Med. 2009; 37:87-93. [PubMed: 19524390]

15. Dominitz JA, Maynard C, Billingsley KG, Boyko EJ. Race, treatment, and survival of veterans with cancer of the distal esophagus and gastric cardia. Med Care. Jan; 2002 40(1 suppl):I14-26. [PubMed: 11789626]

16. Robinson CN, Balentine CJ, Marshall CL, et al. Ethnic disparities are reduced in VA colon cancer patients. Am J Surg. 2010; 200:636-9. [PubMed: 21056144] 
17. Jackson GL, Melton LD, Abbott DH, et al. Quality of nonmetastatic colorectal cancer care in the Department of Veterans Affairs. J Clin Oncol. 2010; 28:3176-81. [PubMed: 20516431]

18. Kussman, MJ. External Peer Review Program (EPRP); Affairs DoV. p. ed2008

19. Hynes DM, Perrin RA, Rappaport S, Stevens JM, Demakis JG. Informatics resources to support health care quality improvement in the veterans health administration. J Am Med Inform Assoc. 2004; 11:344-50. [PubMed: 15187063]

20. [Accessed August 2, 2012] Agency for Healthcare Research and Quality Clinical Classifications Software (CCS) for ICD-9-CM. Available at: http://hcup-us.ahrq.gov/toolssoftware/ccs/ CCSUsersGuide.pdf

21. Biagi JJ, Raphael MJ, Mackillop WJ, Kong W, King WD, Booth CM. Association between time to initiation of adjuvant chemotherapy and survival in colorectal cancer: a systematic review and meta-analysis. JAMA. 2011; 305:2335-42. [PubMed: 21642686]

22. Salz T, Woo H, Starr TD, Jandorf LH, Duhamel KN. Ethnic disparities in colonoscopy use among colorectal cancer survivors: a systematic review. J Cancer Surviv. 2012; 6:372-8. [PubMed: 23054847]

23. Winawer S, Fletcher R, Rex D, et al. Colorectal cancer screening and surveillance: clinical guidelines and rationale—update based on new evidence. Gastroenterology. 2003; 124:544-60. [PubMed: 12557158]

24. Desch CE, Benson AB 3rd, Somerfield MR, et al. Colorectal cancer surveillance: 2005 update of an American Society of Clinical Oncology practice guideline. J Clin Oncol. 2005; 23:8512-9. [PubMed: 16260687]

25. Figueredo A, Rumble RB, Maroun J, et al. Follow-up of patients with curatively resected colorectal cancer: a practice guideline. BMC Cancer. 2003; 3:26. [PubMed: 14529575]

26. National Comprehensive Cancer Network. Clinical Practice Guidelines in Oncology 2003: Colon Cancer. National Comprehensive Cancer Network; Fort Washington, PA: 2003.

27. National Comprehensive Cancer Network. Clinical Practice Guidelines in Oncology 2003: Rectal Cancer. National Comprehensive Cancer Network; Fort Washington, PA: 2003.

28. Rex DK, Kahi CJ, Levin B, et al. Guidelines for colonoscopy surveillance after cancer resection: a consensus update by the American Cancer Society and US Multi-Society Task Force on Colorectal Cancer. CA Cancer J Clin. 2006; 56:160-7. quiz 185-6. [PubMed: 16737948]

29. Cooper GS, Kou TD, Reynolds HL Jr. Receipt of guideline-recommended follow-up in older colorectal cancer survivors: a population-based analysis. Cancer. 2008; 13:2029-37. [PubMed: 18780338]

30. Long MD, Lance T, Robertson D, Kahwati L, Kinsinger L, Fisher DA. Colorectal cancer testing in the national Veterans Health Administration. Dig Dis Sci. 2012; 57:288-93. [PubMed: 21922220]

31. Salz T, Weinberger M, Ayanian JZ, et al. Variation in use of surveillance colonoscopy among colorectal cancer survivors in the United States. BMC Health Serv Res. 2010; 10:256. [PubMed: 20809966]

32. Klabunde CN, Legler JM, Warren JL, Baldwin LM, Schrag D. A refined comorbidity measurement algorithm for claims-based studies of breast, prostate, colorectal, and lung cancer patients. Ann Epidemiol. 2007; 17:584-90. [PubMed: 17531502]

33. Cleves, M.; Gould, W.; Gutierrez, RG.; Marchenko, YV. An Introduction to Survival Analysis Using Stata. 3rd ed. Stata Press; College Station, TX: 2010.

34. Kleinbaum, DG. Survival Analysis - A Self-Learning Text. Springer-Verlag; New York, NY: 1996.

35. Perlin JB, Kolodner RM, Roswell RH. The Veterans Health Administration: quality, value, accountability, and information as transforming strategies for patient-centered care. Am J Manag Care. 2004; 10(11 Pt 2):828-36. [PubMed: 15609736]

36. Bradley CJ, Given CW, Roberts C. Disparities in cancer diagnosis and survival. Cancer. 2001; 91:178-88. [PubMed: 11148575]

37. Pulte D, Redaniel MT, Brenner H, Jeffreys M. Changes in survival by ethnicity of patients with cancer between 1992-1996 and 2002-2006: is the discrepancy decreasing? Ann Onco. 2012; 23:2428-34. 
38. Rabeneck L, Souchek J, El-Serag HB. Survival of colorectal cancer patients hospitalized in the Veterans Affairs Health Care System. Am J Gastroenterol. 2003; 98:1186-92. [PubMed: 12809847]

39. Agha Z, Lofgren RP, VanRuiswyk JV, Layde PM. Are patients at Veterans Affairs medical centers sicker? A comparative analysis of health status and medical resource use. Arch Intern Med. 2000; 160:3252-7. [PubMed: 11088086] 


\section{Clinical Practice Points}

- VA cancer care has been previously examined and it performance is largely considered to be equal to or better than that of the private sector. ${ }^{3,4}$ Historical studies have provided evidence that relative to nonfederal health care systems, racial disparities in CRC care quality may be mitigated in the VA health care system. ${ }^{5-7}$

- We expanded previous work by examining racial differences in 2 dimensions of quality of VA CRC care: processes (time to treatment) and outcomes (survival). We identified no evidence of clinically meaningful racial differences in time from surgery to initiation of chemotherapy, surveillance colonoscopy, or death. Moreover, care was administered within acceptable time frames. Median time from surgery to adjuvant chemotherapy was approximately 7 weeks.

- During the time that these patients were treated (2003-2006), the generally accepted goal was to administer chemotherapy within 6 to 12 weeks postoperatively. Similarly, the median time to colonoscopy was approximately 368 days. This is very close to the 1-year goal (ie, 365 days).

- Although access to care and causality were not examined as part of our study, these data seem to suggest that when patients are given equal access to care, their outcomes can be comparable. Moreover, these findings highlight the importance of clinicians' adherence to clinical practice guidelines and timeliness recommendations.

- Clinicians play a critical role in guaranteeing that patients receive proper care at appropriate time intervals. 


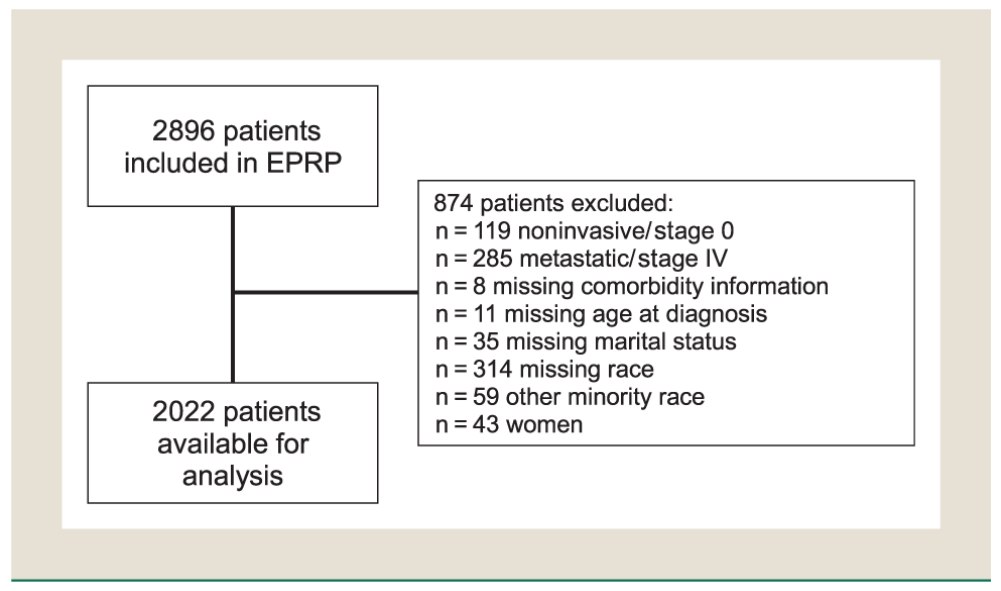

Figure 1.

Colorectal Cancer Cohort Assembly 
Table 1

Description of CRC Patient Cohort and Key Variables

\begin{tabular}{|c|c|c|c|}
\hline Variable & $\begin{array}{c}\text { White Patients } \\
(\mathrm{n}=1712) \%(\mathrm{n}) \text { or } \\
\text { Mean (SD) }\end{array}$ & $\begin{array}{l}\text { African American Patients } \\
(\mathrm{n}=\mathbf{3 1 0}) \%(\mathrm{n}) \text { or } \\
\text { Mean }(\mathrm{SD})\end{array}$ & $\begin{array}{c}\text { Full Sample } \\
(\mathrm{n}=\mathbf{2 0 2 2}) \%(\mathrm{n}) \text { or } \\
\text { Mean }(\text { SD) }\end{array}$ \\
\hline \multicolumn{4}{|l|}{ Dependent Variables } \\
\hline Surgery to adjuvant 5-FU chemotherapy (d) & $63.5(55.1)$ & $65.6(45.6)$ & $63.8(53.6)$ \\
\hline Surgery to surveillance colonoscopy (d) ${ }^{a}$ & $391.9(195.4)$ & $420.4(221.8)$ & $396.0(199.6)$ \\
\hline Surgery to death (d) & $1136.6(730.4)$ & $988.0(1258.6)$ & $1112.9(838.1)$ \\
\hline \multicolumn{4}{|l|}{ Independent Variable } \\
\hline White race & $100 \%(1712)$ & $0 \%(0)$ & $84.7 \%(1712)$ \\
\hline \multicolumn{4}{|l|}{ Other Control Variables } \\
\hline \multicolumn{4}{|l|}{ Age at diagnosis } \\
\hline$<55$ years & $8.1 \%(138)$ & $13.6 \%(42)$ & $8.9 \%(180)$ \\
\hline $55-64$ years & $30.5 \%(522)$ & $30.0 \%(93)$ & $30.4 \%(615)$ \\
\hline $65-74$ years & $28.7 \%(492)$ & $27.1 \%(84)$ & $28.5 \%(576)$ \\
\hline $75+$ years & $32.7 \%(560)$ & $29.4 \%(91)$ & $32.2 \%(651)$ \\
\hline Married & $53.9 \%(922)$ & $39.7 \%(123)$ & $51.7 \%(1045)$ \\
\hline \multicolumn{4}{|l|}{ Region of United States } \\
\hline South & $34.2 \%(586)$ & $56.1 \%(174)$ & $37.6 \%(760)$ \\
\hline North & $19.7 \%(337)$ & $15.8 \%(49)$ & $19.1 \%(386)$ \\
\hline Central & $22.7 \%(388)$ & $20.3 \%(63)$ & $22.3 \%(451)$ \\
\hline West & $23.4 \%(401)$ & $7.8 \%(24)$ & $21.0 \%(425)$ \\
\hline \multicolumn{4}{|l|}{ Stage at diagnosis } \\
\hline Stage I & $30.3 \%(518)$ & $30.3 \%(94)$ & $30.3 \%(612)$ \\
\hline Stage II & $38.4 \%(657)$ & $32.3 \%(100)$ & $37.4 \%(757)$ \\
\hline Stage III & $31.4 \%(537)$ & $37.4 \%(116)$ & $32.3 \%(653)$ \\
\hline NCI Combined Comorbidity Score & $0.28(0.43)$ & $0.25(0.40)$ & $0.27(0.43)$ \\
\hline
\end{tabular}

Abbreviations: $\mathrm{NCI}=$ National Cancer Institute $\mathrm{SD}=$ standard deviation .

${ }^{a}$ The sample size is larger in the Cox regression models because colonoscopies occurring outside of this time window are included as failures 
Table 2

Cox Proportional Hazard Model Regression Results

\begin{tabular}{|c|c|c|c|c|c|c|c|c|c|}
\hline \multirow{3}{*}{ Demographic Characteristics } & \multicolumn{3}{|c|}{ Surgery to Chemotherapy $a$} & \multicolumn{3}{|c|}{ Surgery to Colonoscopy $b$} & \multicolumn{3}{|c|}{ Surgery to Death $c$} \\
\hline & HR & $95 \% \mathrm{CI}$ & $P$ Value & HR & $95 \% \mathrm{CI}$ & $P$ Value & HR & $95 \% \mathrm{CI}$ & $P$ Value \\
\hline & & & & & & & & & \\
\hline White & 0.82 & $0.38-1.76$ & .61 & 0.63 & $0.43-0.94$ & $.02 *$ & 0.94 & $0.79-1.12$ & .49 \\
\hline Married & 0.70 & $0.35-1.39$ & .30 & 1.28 & $0.91-1.78$ & .15 & 0.97 & $0.86-1.10$ & .66 \\
\hline Age at Diagnosis & & & & & & & & & \\
\hline$<55$ years & 2.78 & $0.71-10.94$ & .14 & 1.58 & $0.89-2.81$ & .12 & 0.76 & $0.56-0.99$ & $.04^{*}$ \\
\hline 55-64 years & 2.36 & $0.66-8.36$ & .18 & 1.28 & $0.82-1.99$ & .27 & 0.99 & $0.84-1.18$ & .96 \\
\hline $65-74$ years & 1.76 & $0.46-6.70$ & .41 & 1.60 & $1.03-2.47$ & $.04^{*}$ & 0.90 & $0.77-1.06$ & .21 \\
\hline $75+$ years $($ referenc & & & & & & & & & \\
\hline Region of United Sta & & & & & & & & & \\
\hline North & 0.06 & $0.01-0.49$ & $.01^{*}$ & 0.90 & $0.56-1.44$ & .65 & 1.10 & $0.92-1.33$ & .29 \\
\hline Central & 0.33 & $0.11-0.97$ & $.04^{*}$ & 1.84 & $1.23-2.76$ & $.00^{*}$ & 0.99 & $0.84-1.18$ & .96 \\
\hline West & 0.39 & $0.15-0.99$ & .05 & 1.13 & $0.68-1.87$ & .64 & 1.02 & $0.86-1.21$ & .85 \\
\hline South (reference) & & & & & & & & & \\
\hline NCI Combined & & & & & & & & & \\
\hline Comorbidity Score & 1.69 & $0.68-4.19$ & .26 & 1.44 & $0.92-2.26$ & .11 & 1.01 & $0.89-1.15$ & .83 \\
\hline Stage at Diagnosis & & & & & & & & & \\
\hline Stage I & - & - & - & 1.17 & $0.81-1.69$ & .41 & 0.84 & 0.71-0.99 & $.04^{*}$ \\
\hline Stage II (reference) & & & & & & & & & \\
\hline Stage III & - & - & - & 0.85 & $0.55-1.32$ & .48 & 1.32 & $1.13-1.53$ & $.00 *$ \\
\hline $\mathbf{N}$ & 632 & & & 1083 & & & 991 & & \\
\hline
\end{tabular}

-Indicates that stage was not included in the surgery to chemotherapy timeliness measure because analysis was limited to patients with stage II and stage III disease.

Abbreviations: $\mathrm{CI}=$ confidence interval; $\mathrm{HR}=$ hazard ratio; $\mathrm{NCI}=$ National Cancer Institute.

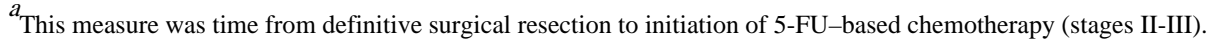

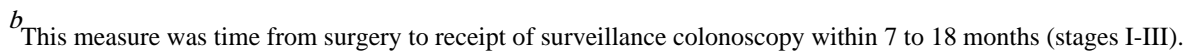

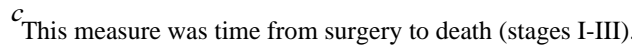

Indicates statistical significance at the $<.05$ alpha level. 